\title{
A novel technique for ventral orbital stabilization: the masseter muscle flap
}

Amilan Sivagurunathan ${ }^{1}$ BVSc, MMedVet (Ophth) Sonja C. Boy ${ }^{2}$ BChD, MChD(Oral Path), PhD and Gerhard Steenkamp ${ }^{1}$ BSc, BVSc, MSc.

${ }^{1}$ Section of Surgery, Department of Companion Animal Clinical Studies, Faculty of Veterinary Science, University of Pretoria, South Africa

${ }^{2}$ Department of Oral Pathology and Oral Biology, School of Dentistry, University of Pretoria, Pretoria, South Africa

Address Communications to:

Gerhard Steenkamp

Private Bag X04

Onderstepoort

0110

South Africa

Tel: +27 125298276

Fax: +27 125298479

e-mail: gerhard.steenkamp@up.ac.za

\section{Running Title:}

Ventral orbit stabilization using a masseter flap 


\section{Abstract}

Loss of the caudal maxilla and ventral orbit after tumor resections can have negative functional and aesthetic influences on the eye involved. This article reports on a case of a caudal maxillary acanthomatous ameloblastoma involving the ventral orbit that was resected and stabilized with a masseter muscle flap. The masseter muscle flap was generated from the superficial belly of the masseter muscle in order to close a defect in the orbital rim, created by a caudal maxillectomy. None of the published complications such as enophthalmos, excessive lacrimation, globe deviation or strabismus were noted, eight months following the procedure. The only clinical sign present at the time of re-evaluation was mild lacrimation. The authors propose the use of a masseter muscle flap as a viable technique in stabilizing the ventral orbit after caudal maxillectomy and ventral orbitectomy, preventing the complications associated with this surgery.

Key words: dog, maxillectomy, orbit stabilization, flap reconstruction, acanthomatous ameloblastoma, strabismus

\section{Introduction}

The maxilla provides support to structures of the mid-face including the globe, eyelids, cheeks, nose and lips. It is also the principal bony structure of the hard palate. Tumors that frequently involve the maxilla and rostral skull of the dog include fibrosarcoma, osteosarcoma, squamous cell carcinoma, malignant melanoma, and acanthomatous ameloblastoma. ${ }^{(1,2)}$ Most often, these tumors are caudally positioned, invasive and complex, involving critical adjacent structures such as the orbit, nose and mouth with difficult surgical access and poorer treatment success and prognosis. ${ }^{(1,2)}$ 
The canine orbital margin is incomplete. A well-developed orbital ligament completes it caudolaterally, originating from the zygomatic process of the frontal bone and inserts on the frontal process of the zygomatic bone. ${ }^{(3)}$ Anteriorly the globe is supported mainly by peri-orbital connective tissues while more posteriorly soft tissue consisting of $\mathrm{Mm}$. temporalis and pterygoideus, glandular parenchyma from the zygomatic salivary gland and ample retrobulbar fat are responsible for support. ${ }^{(3)}$ Management of caudally situated neoplasms commonly necessitates wide surgical resection such as caudal maxillectomy and partial orbitectomy to ensure tumor free margins and prevent tumor recurrence. These procedures may however compromise orbital stability ${ }^{(4)}$ and lead to complications such as traumatic uveitis, optic nerve damage, hyphaema, globe rupture, retinal detachment, enophthalmos, strabismus, chronic epiphora, nasolacrimal duct damage and conjunctivitis all which influence function and cosmesis. ${ }^{(5-7)}$ Ventral orbital defects as a result of surgical procedures or even extensive trauma may be challenging to repair, even in humans. ${ }^{(8)}$

Due to the proximity of the masseter muscle to the caudal maxilla and ventral orbital floor it has already been used in humansto stabilize the ventral orbit. ${ }^{(9,10)}$ The masseter muscle consists of superficial, middle and deep layers that are covered by a strong glistening aponeurosis and is positioned laterally on the ramus of the mandible of the dog. Of the three muscle layers, the superficial muscle layer is the largest and strongest. It originates mainly from the ventral border of the rostral half of the zygomatic arch and passes caudoventrally to insert on the ventromedial surface of the mandible, as well as on a tendinous raphe between the masseter and the medial pterygoid muscles. Part of the deep muscle layer intermingles with temporal muscle before inserting on the rostral ridge of the masseteric fossa. $^{(11)}$ The superficial arterial blood supply to the masseter muscle is derived primarily from the transverse facial artery while the deeper muscle layers are supplied by the maxillary artery and the facial artery ${ }^{(12)}$. 
The use of a masseter muscle flap to reconstruct defects and stabilize the ventral orbit has not been reported in the veterinary literature. This paper details a reconstructive technique by means of a masseter muscle flap following caudal maxillectomy and partial orbitectomy using a combined

intraoral and dorsolateral approach. ${ }^{(4)}$ The procedure was developed whilst planning surgery for a clinical case of a dog presenting with a large caudal maxillary neoplasm. Two detailed cadaver studies were performed after which the procedure was applied to the dog with a maxillary neoplasm.

\section{Materials and Methods}

Two dolicocephalic cadavers euthanized for reasons unrelated to this study were used for the experimental component of this study. The owners consented to elective post mortems and/or using their dogs for training purposes at the Onderstepoort Veterinary Academic Hospital of the University of Pretoria.

The dogs were positioned in left lateral recumbency in order to simulate the clinical case where the neoplasm was situated in the right maxilla. The combined intra-and extraoral technique of Lascelles was used to expose the caudal maxilla. ${ }^{(4)}$ Thereafter the periosteum of the zygomatic bone was incised $2 \mathrm{~mm}$ ventral to the orbital rim and the incision extended from the medial canthus (angulis oculi medialis) to $1 \mathrm{~cm}$ beyond the frontal process where the orbital ligament attaches. The periosteum was elevated dorsally and ventrally (Fig. 1a) using a Meads periosteal elevator. The masseter muscle was subsequently cut at its attachment to the ventral surface of the zygomatic arch (Fig. 1b). After exposing the zygomatic bone the periosteal incision was extended to the level of the infra-orbital foramen to expose the maxillary bone. A $2.4 \mathrm{~cm}$ ostectomy of the zygomatic arch was done extending from the maxilla-zygomatic interface caudally to the orbital ligament excluding the latter (Fig. 1c) using a round tungsten carbide burr on a Dremmel tool. This defect size was planned pre-operatively on computed tomography (CT) scans of the clinical case. 

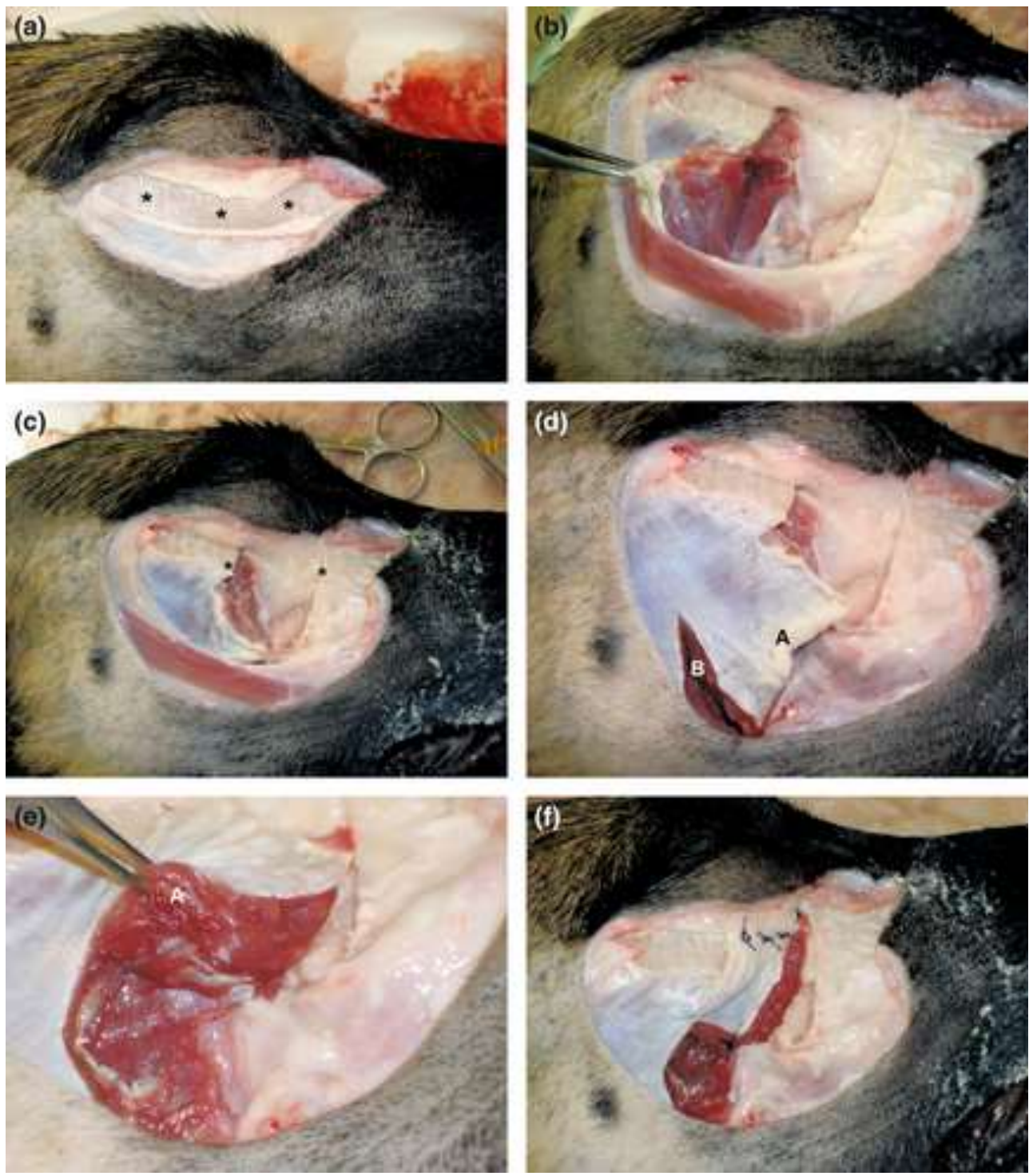

Figure 1a. A periosteal incision was made $2 \mathrm{~mm}$ ventral to the dorsal rim of the zygomatic arch (asterisks) and the periosteum stripped with a Meads periosteal elevator. Figure 1b. The rostro-dorsal attachment of the masseter muscle was cut free from its origin, on the ventral surface of the zygomatic arch. Figure 1c. A defect of $2.4 \mathrm{~cm}$ was created in the zygomatic arch consistent with the anticipated defect to be created in our clinical case. The zygomatic arch osteotomy lines are indicated by the black asterisks $(*)$. Figure 1d. The $2.4 \mathrm{~cm}$ distance was measured (A) from the dorsal to ventral rostral border of the masseter muscle, enough tissue to cover the defect. A $2 \mathrm{~cm}$ incision was then made in a rostro-caudal direction (B) to free the muscle tissue. Figure 1e. Care was taken to bluntly dissect the superficial belly of the masseter muscle (A) free. The remainder of the muscle was left attached to maintain good blood supply. Figure 1f. The masseter muscle flap was rotated into the defect and anchored with 3 sutures to the periosteum which covered the dorsal rim of the zygomatic arch. One suture was placed at the ventral border of the flap in order to prevent the flap from lifting. 
A $2 \mathrm{~cm}$ rostro-caudal incision was made in the superficial belly of the masseter muscle, $2.4 \mathrm{~cm}$ ventral to its zygomatic attachment in order to create a masseter flap of sufficient length to close the bony defect created (Fig 1d). Gentle blunt dissection was used to free the ventral part of the flap. The blunt dissection was not done on the dorsal $1 \mathrm{~cm}$ of the superficial masseter muscle belly, as this was left attached to the deep belly in order to maximize blood supply (Fig 1e). The free part of the muscle was rotated into place and sutured to the periosteum elevated from the orbital rim (Fig 1f). The most rostral suture was placed in the periosteum of the maxillary bone. A single suture was placed at the most ventral part of the free flap, attaching it to the deep belly (Fig 1f).

\section{Clinical case}

A 12 year old, intact male cross breed dog $(20 \mathrm{~kg})$ was referred and admitted for regrowth of a caudal right maxillary mass extending from the fourth maxillary premolar (tooth 104) caudally to the last molar (tooth 110) (Fig 2). The mass was allegedly removed six months prior from the same

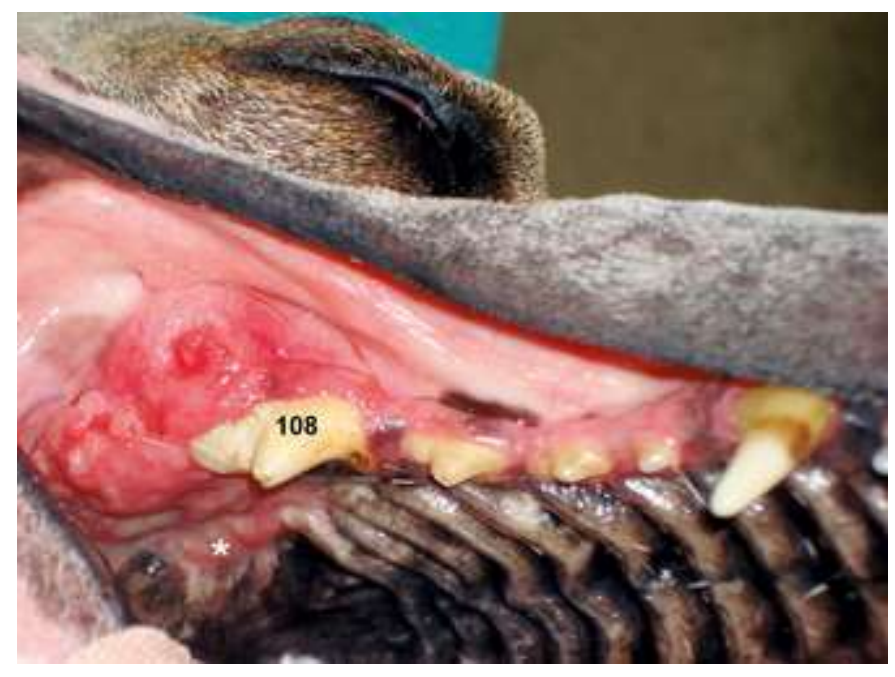

Figure 2. Intraorally the mass extendend from the caudal root of the maxillary $4{ }^{\text {th }}$ premolar tooth (108) to just caudal of the maxillary $2^{\text {nd }}$ molar (110). The mass also infiltrated the mucosal lining of the hard palate (white *). 
location and was diagnosed as a fibrosarcoma at a different laboratory. No adjuvant treatment was given. On physical examination, the dog was bright, alert and otherwise normal. The mandibular, parotid and cranial cervical lymph nodes were not enlarged on palpation. Fine needle aspirations (FNA) of the above lymph nodes were normal. Hematologic and biochemical analysis were within normal limits. Ocular examination revealed mild to moderate exophthalmos of the right globe and bilateral nuclear sclerosis. The globe could not be retropulsed, and pain was absent on opening the jaw. Menace response was positive in both eyes (OU). Direct and indirect pupillary light (PLRs) and dazzle reflexes were positive OU. Tear production, as assessed by Schimmer Tear Test-1 (STT) (ScheringPlough Animal Health, Union, NJ) was within normal range OU. Using slit-lamp biomicroscopy the corneas appeared normal OU. Oral examination revealed absent right maxillary molars (teeth 109-110) with adjacent hard palate distortion from the oral mass (Fig 2). The oral mass was noticed by the owner during the past 2 months with exophthalmos only present the last 2 weeks.

A CT scan of the skull and thorax was performed with the dog anaesthetized, to further assess and characterize the margins of the mass and possible metastases to the lymph nodes and lungs. The CT study revealed a localized bone destroying mass involving the caudal maxilla and rostral zygoma. This lesion extended from the level of the first molar (tooth 109) caudally along the zygomatic bone to just rostral of the orbital ligament (Fig 3a). Mild to moderate exophthalmos of the right globe was noted. The mass caused focal destruction of the adjacent infra-orbital canal (Fig 3b). The temporomandibular joint and coronoid process of the mandible as well as the maxillary recess and the nasal cavity on the right side appeared unaffected. No signs of metastasis to the lymph nodes, brain, or thorax were noticed on CT. A surgical biopsy of the oral mass was performed, which was diagnosed as an acanthomatous ameloblastoma.

Three days later, the dog was induced with $1 \%$ Propofol $(6 \mathrm{mg} / \mathrm{kg}$ [IV]), anaesthetized with Isoflurane (1.5\%-2\%), positioned in ventral recumbency, and skin on the right side of the head 

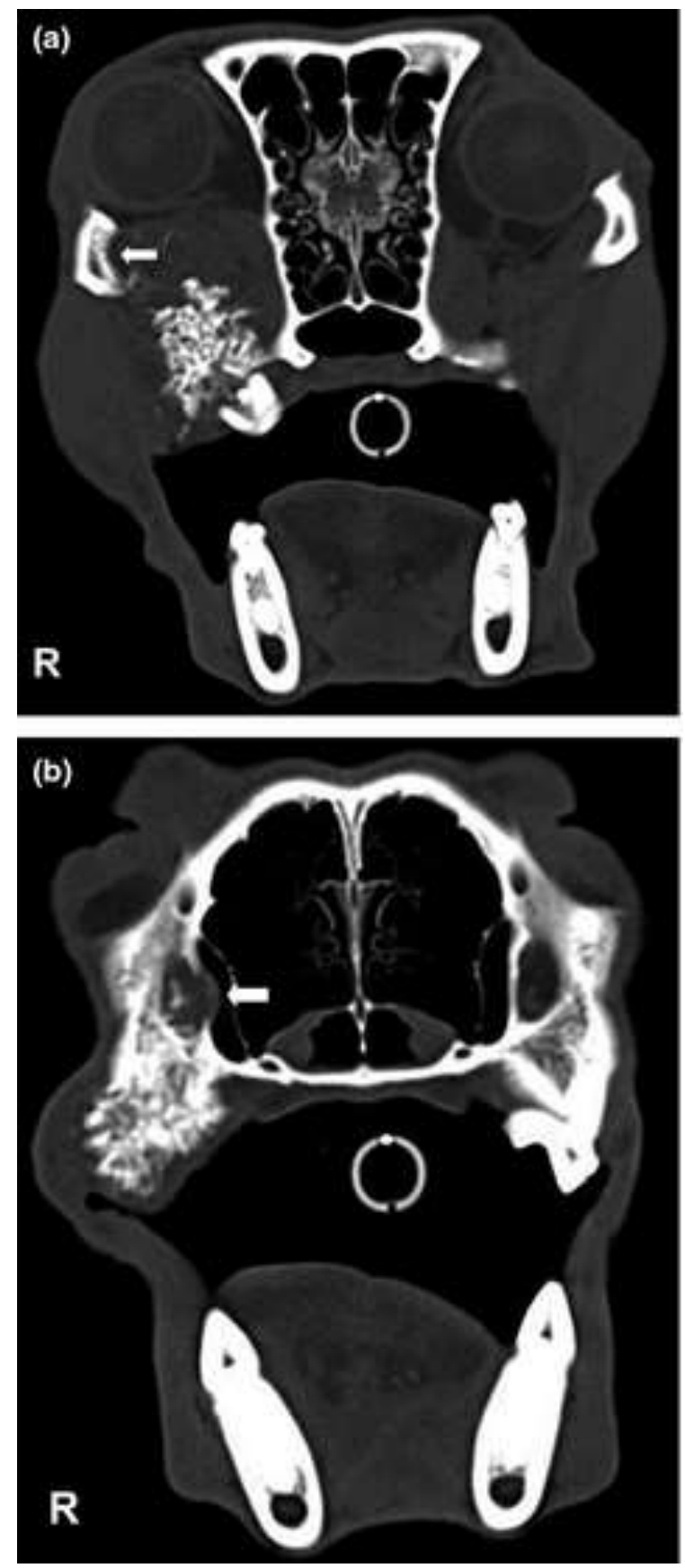

Figure 3a. This is a transverse CT image viewed in a bony window ww:wl (1500/450) of the mass extending caudally, destroying the zygomatic arch (white arrow) and causing displacement of the eye (exophthalmos). The latter was as a result of the mass extending into the ventral orbit. Figure 3b. This is a transverse CT image viewed in a bony window ww:wl (1500/450) showing the rostral extension of the mass. The mass extended into the infraorbital canal (white arrow). 
aseptically prepared. Cefazolin $(20 \mathrm{mg} / \mathrm{kg})$ was administered IV at anesthetic induction and continued for 4 days post operatively (20mg/kg IV every 6 hours). For analgesia a MLK (Morphine Lignocaine-Ketamine 0.24/0.3/0.06mg/kg/hr [IV]) combination infusion was adminstered at $2 \mathrm{ml} / \mathrm{kg} / \mathrm{hr}$ postoperatively for 48 hours. Ropivicaine ( $2 \mathrm{ml}$ ) was infiltrated into the infra-orbital foramen, at the periphery of the mass on the hard palate and within the right buccal mucosa ventral to the orbit at $0.2 \mathrm{ml}$ aliquotes. The tumour was approached through an intra- and extra-oral technique. ${ }^{(4)}$ As far as possible the authors maintained a $1 \mathrm{~cm}$ free margin all around the tumour. The maxilla was paramedian of the palate, rostrally to the diastema between the $2^{\text {nd }}$ and $3^{\text {rd }}$ maxillary premolars (teeth 106 and 107), dorsally to the infra-orbital canal up to the level of the medial orbital rim and extending caudally up to the rostral margin of the orbital ligament. Once the bone was resected, it was placed in $10 \%$ buffered formalin for margin evaluation. The masseter muscle flap was generated as described above and sutured into place using a monofilament absorbable material (Monocryl@ 4/0). The rest of the skin was closed in a 3 layer fashion using the same material. Intra-orally the buccal mucosa was mobilized by blunt dissection and sutured to the palatal mucosa using single interrupted sutures (Monocryl@ 4/0).

The patient recovered uneventfully in the intensive care unit and was eating a soft liquidized diet (Hills a/d) within 12 hours of the surgery. Three days post-operative, the dog was discharged and follow-up examination visits was schedule for 1 week, 1 month and 3 months post-operative. Apart from a mild exotropia that remained for 2 weeks postoperative, before the globe returned to its normal position, no other comlications was seen or reported by the owner.

At all these follow-up examinations the dog was doing well with no complications seen or reported by the owner.

Eight months post-operatively a follow up CT scan was performed to evaluate the ostectomy site. Linear new bone formation was observed at the level of the ventral orbit, along the dorsal border of the superficial masseter muscle flap (Fig. 4). The new bone extended medially from the zygomatic arch in 

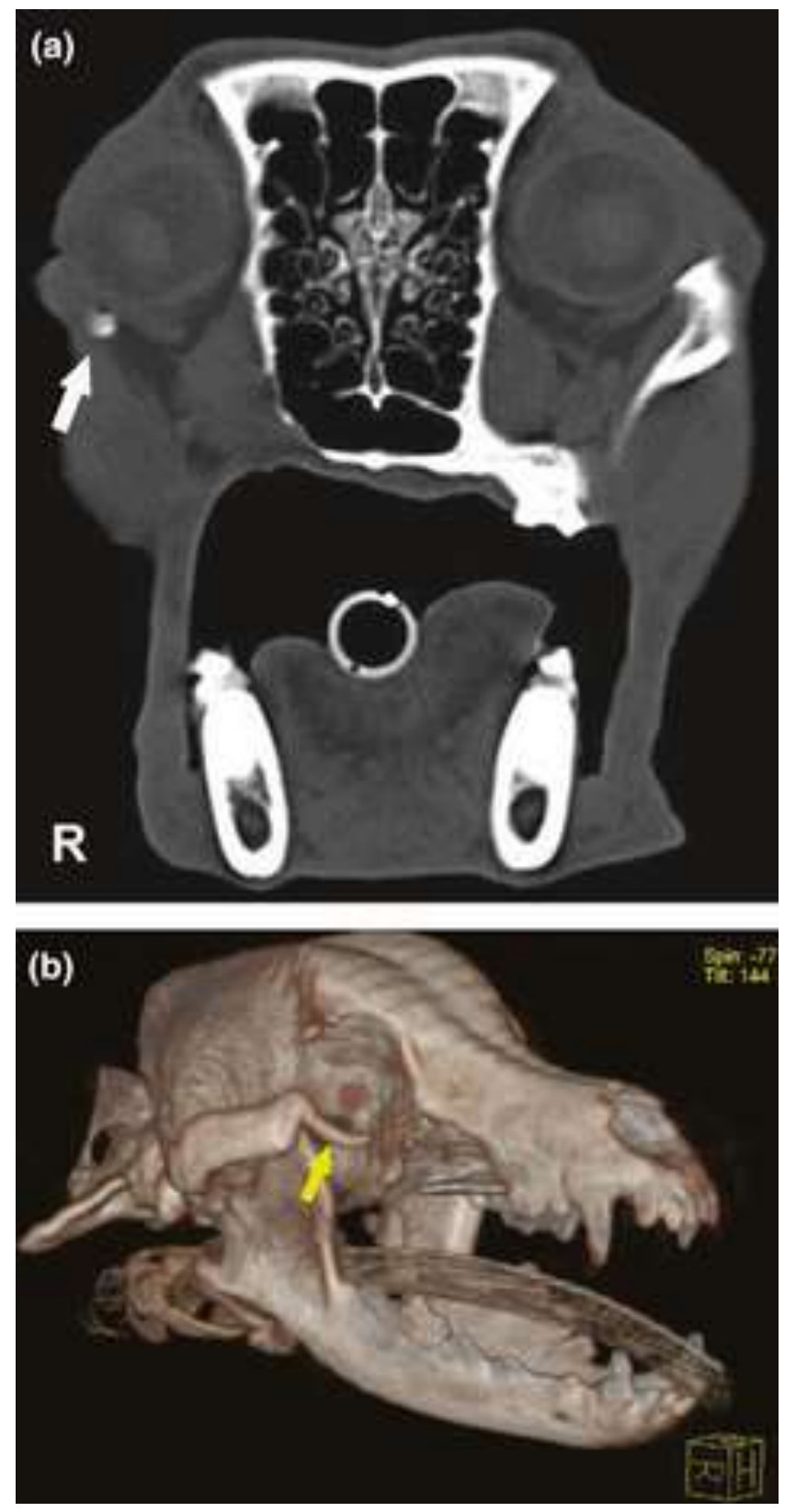

Figure 4a. On CT examination 8 months post-operative no tumour regrowth was visible. This transverse CT image viewed in a bony window ww:wl (1500/450) shows the bone formation present in the area, ventral to the eye (arrow), where the periosteum of the resected zygomatic arch was saved. Figure 4b. A VRT 3D image viewed in a scapula algorithm of the right lateral skull of the dog, 8 months post-operative showing no tumour regrowth and new bone formation ventral to the orbit (arrow). 


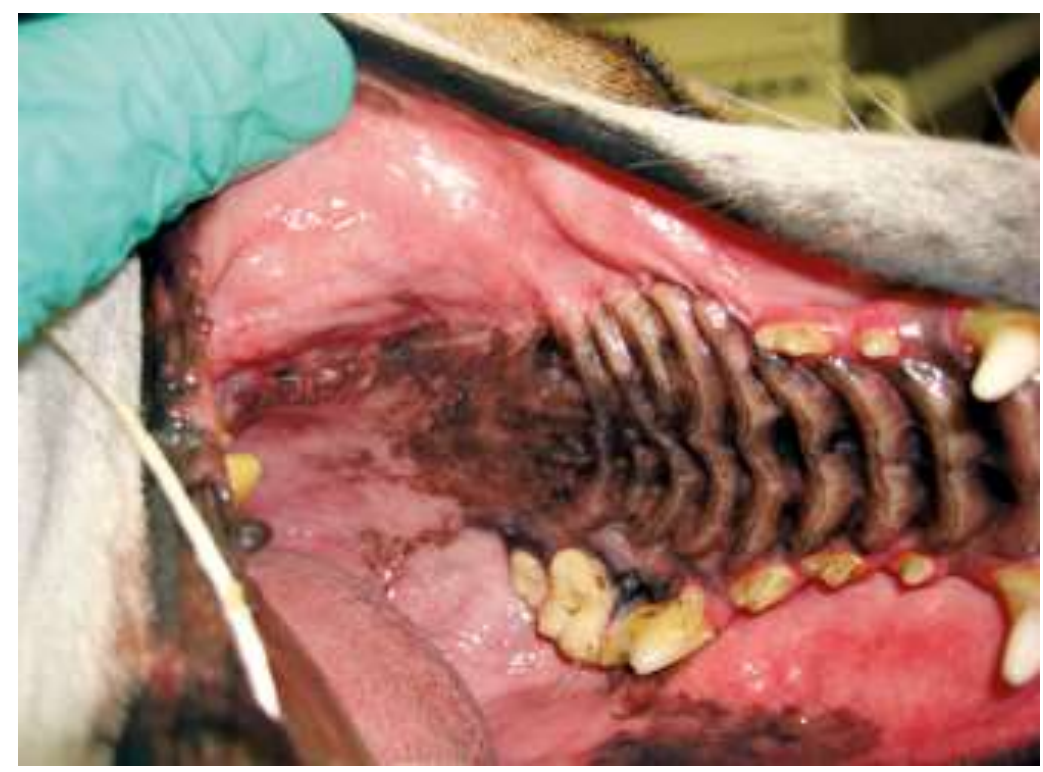

Figure 5. Intraoral view of the dog at the 8 month post-operative evaluation. No dehiscence of the wound, or regrowth of the tumour was evident.

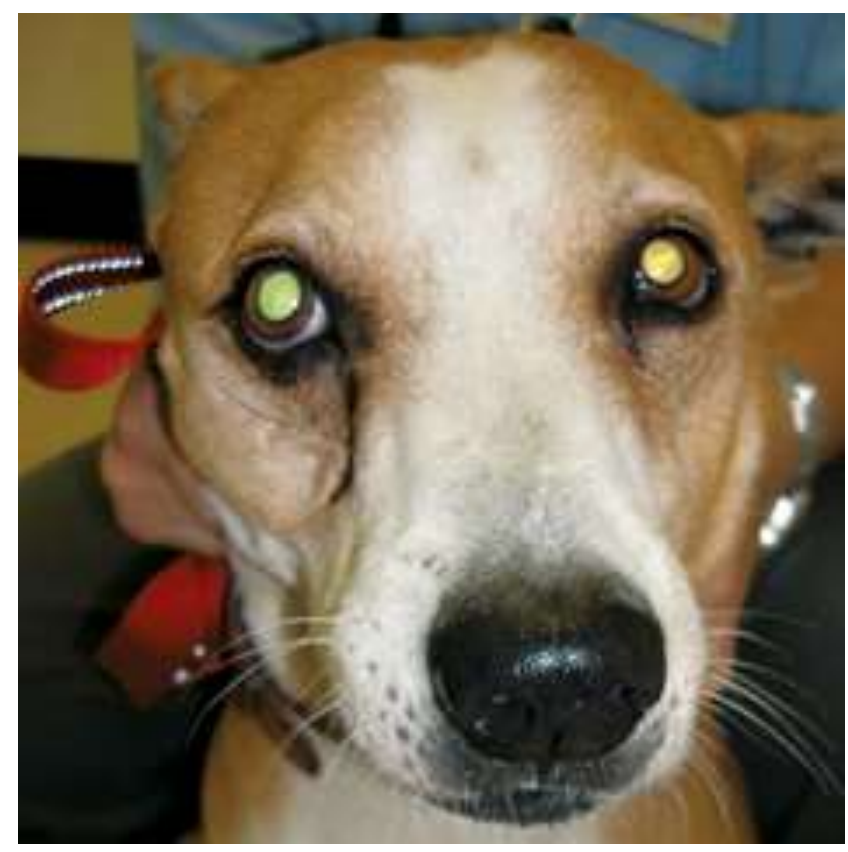

Figure 6. Apart from mild lacrimation, no other ocular abnormalities were present in the dog at the 8 month post-operative evaluation. There is mild asymmetry of the face with a slight bulging of the right hand side. The authors ascribe this due to post-operative scar tissue formation exacerbated by the defect created by removing part of the maxillary bone. 
the direction of the medial margin of the orbit. Intra-orally the surgical site healed completely with no evidence of soft tissue regrowth (Fig.5). At this time no ocular complications were seen apart from mild lacrimation of the right eye (Fig 6). The dog's mouth had the full range of motion as before the surgery, and the owner had no complaints regarding the dog's use of his jaws.

\section{Discussion}

When large orbital floor defects are resected, support to the orbital contents need to be reestablished otherwise the globe will sink ventrally. In humans these defects can result in significant diplopia, dystopia and functional loss of the eye ${ }^{(8)}$. Traditionally in human maxillofacial surgery, large maxillectomy defects have been reconstructed using bone grafts (iliac crest, cranium, and rib), mesh implants, local and pedicle flaps such as cheek flap, temporalis flap, sternomastoid-platysma cutaneous flap, radial forearm flap, scapular flap and latissimus dorsi flap ${ }^{(13-17)}$. The choice of techniques is largely influenced by the surgeon's preference. The masseter flap described by Conley and Gulane (1978) has proved to be bulky enough to fill a large ventral orbital defect ${ }^{(10)}$. In addition, it is a safe, one stage procedure, which does not require elaborate technique or aftercare and does not result in aesthetic and visual loss. Tumours of the maxillo-facial area frequently involve the masseter muscle by direct infiltration thereof. This may restricts its use as a muscle flap in reconstructive surgery. If the tumor is extensive, alternative reconstructive methods should be used ${ }^{(10)}$. The masseteric branches of the transverse facial artery and internal maxillary artery supply the dorsally based masseter muscle flap and must be carefully preserved to minimize post-operative contraction of the flap. In the process of creating a superficial axial muscle flap, the following basic principles of flaps should also be followed. Firstly the muscle flap should generously fit the defect. Secondly the ratio of length to width of the flap should be 2:1. Thirdly, avoid kinking, compression, tension or severe angulations and always use 2 layers of sutures for positioning and support. 
In terms of flap positioning and mobility, the aponeurosis of the superficial masseter muscle flap lacks great flexibility, limiting its use in the correction of various mid-face defects. An island based masseter muscle flap is defined as a flap of the masseter muscle pivoting around its own blood supply). The superiorly based masseter muscle flap is defined as a flap where the origin of the muscle is kept as the pivot. Due to its flexibility in reaching anatomically difficult sites, pliability for larger defects and usual absence of postoperative trismus as complication ${ }^{(10)}$ the superiorly based masseter muscle flap is preferred above island-based masseter flaps to correct oropharyngeal defects.

The masseter muscle flap not only stabilized the ventral orbit of the globe, but also created scaffolding for osteogenesis and new periosteal bone formation. The masseter muscle flap in itself was free of calcification and was minimally atrophied, due to careful preservation of the deep and superficial vascular supply.

Haemorrhage and wound dehiscence have been well described as common complications intra-and post-operatively when performing caudal maxillectomies. For this single case report, blood loss although present intra-operatively was considered minimal and there was no post-operative wound dehiscence.

Damage to the nasolacrimal duct can occur during resection of the caudal maxilla and may explain the presence of mild lacrimation postoperatively (Fig 6). Decreased interincisal distance has been reported in the human literature after the use of a superiorly based masseter muscle flap ${ }^{(10)}$. No restriction of jaw movement was seen in the dog. This could be due to the fact that the flap employed here had a different orientation and was constructed of only the superficial belly of this muscle. Before performing the caudal maxillectomy, the right globe had deviated dorso-laterally and rostrally due to the absence of osseous support in the caudo-lateral orbital margin. Hence displacement forces ventral to the globe, from the caudal maxillary mass had inevitably created a moderate exophthalmos. Within 
twenty four hours postoperatively, the exopthalmos had reduced, allowing normal blinking reflexes and palpebral closure.

The slight asymmetry seen on the right hand-side of the face (Fig. 6) is ascribed to post-operative scar tissue formation in this area. The asymmetry is however exacerbated by the fact that the zygomatic process of the maxillary bone as well as some maxillary bone was resected. There is therefore not a gradual transition from maxilla to zygomatic arch as on the left hand-side.

\section{References:}

1. Hoyt R, Withrow SJ. Oral malignancy in the dog. Journal of the American Animal Hospital Association 1984: 83-90.

2. Todoroff RJ, Brodey RS. Oral and pharyngeal neoplasia in the dog: a retrospective survey of 361 cases. Journal of the American Veterinary Medical Association 1979; 175: 567-71.

3. Murphy CJ, Pollock RVH. The Eye. In: Evans HE, editor. Miller's Anatomy of the Dog. 3rd ed. Philadelphia: W.B. Saunders Company. 1993; 1009 - 57.

4. Lascelles BD, Thomson MJ, Dernell WS, et al. Combined dorsolateral and intraoral approach for the resection of tumors of the maxilla in the dog. Journal of the American Animal Hospital Association 2003; 39: 294-305.

5. Matthiesen DT, Manfra Marretta S. Results and complications associated with partial mandibulectomy and maxillectomy techniques. Problems in Veterinary Medicine 1990; 2: 248-75.

6. Konrade KA, Clode AB, Michau TM, et al. Surgical correction of severe strabismus and enophthalmos secondary to zygomatic arch fracture in a dog. Veterinary Ophthalmology 2009; 12: 11924.

7. O'Brien MG, Withrow SJ, Straw RC, et al. Total and partial orbitectomy for the treatment of periorbital tumors in 24 dogs and 6 cats: a retrospective study. Veterinary Surgery 1996; 25: 471-9. 
8. Cordeiro PG, Disa JJ. Challenges in midface reconstruction. Seminars in Surgical Oncology 2000; 19: 218-25.

9. Conley J, Gullane PJ. The masseter muscle flap. Laryngoscope 1978; 88: 605-12.

10. Antoniades K, Lasaridis N, Vahtsevanos K, et al. Superiorly based and island masseter muscle flaps for repairing oropharyngeal defects. Journal of Craniomaxillofacial Surgery 2005; 33: 334-9.

11. Hermanson JW, Evans HE. The Muscular System. In: Evans HE, editor. Miller's Anatomy of the Dog. 3rd ed. Philadelphia: W.B. Saunders company. 1993; 280-1.

12. Evans HE. The Heart and Arteries. In: Evans HE, editor. Miller's Anatomy of the Dog. 3rd ed. Philadelphia: W.B. Saunders Co. 1993; 602-19.

13. Cordeiro PG, Santamaria E, Kraus DH, et al. Reconstruction of total maxillectomy defects with preservation of the orbital contents. Plastic and Reconstructive Surgery 1998; 102: 1874-84; discussion $85-7$.

14. Schubert W, Gear AJ, Lee C, et al. Incorporation of titanium mesh in orbital and midface reconstruction. Plastic and Reconstructive Surgery 2002; 110: 1022-30; discussion 31-2.

15. Boahene KD. Dynamic muscle transfer in facial reanimation. Facial Plastic Surgery 2008; 24 : 204-10.

16. Cordeiro PG, Santamaria E. Primary reconstruction of complex midfacial defects with combined lip-switch procedures and free flaps. Plastic and Reconstructive Surgery 1999; 103: 1850-6.

17. Duffy FJ, Jr., Gan BS, Israeli D, et al. Use of bilateral folded radial forearm free flaps for reconstruction of a midface gunshot wound. Journal of Reconstructive Microsurgery 1998; 14: 89-96. 